\title{
An Optimized Damage Identification Method of Beam Using Wavelet and Neural Network
}

\author{
Bingrong Miao*, Mingyue Wang, Shuwang Yang, Yaoxiang Luo, Caijin Yang \\ State Key Laboratory of Traction Power, Southwest Jiaotong University, Chengdu, China \\ Email: ^brmiao@home.swjtu.edu.cn
}

How to cite this paper: Miao, B.R., Wang, M.Y., Yang, S.W., Luo, Y.X. and Yang, C.J. (2020) An Optimized Damage Identification Method of Beam Using Wavelet and Neural Network. Engineering, 12, 748-765. https://doi.org/10.4236/eng.2020.1210053

Received: September 21, 2020

Accepted: October 23, 2020

Published: October 26, 2020

Copyright $\odot 2020$ by author(s) and Scientific Research Publishing Inc. This work is licensed under the Creative Commons Attribution International License (CC BY 4.0).

http://creativecommons.org/licenses/by/4.0/

(c) (i) Open Access

\begin{abstract}
An optimized damage identification method of beam combined wavelet with neural network is presented in an attempt to improve the calculation iterative speed and accuracy damage identification. The mathematical model is developed to identify the structure damage based on the theory of finite elements and rotation modal parameters. The model is integrated with BP neural network optimization approach which utilizes the Genetic algorithm optimization method. The structural rotation modal parameters are performed with the continuous wavelet transform through the Mexico hat wavelet. The location of structure damage is identified by the maximum of wavelet coefficients. Then, the multi-scale wavelet coefficients modulus maxima are used as the inputs of the BP neural network, and through training and updating the optimal weight and threshold value to obtain the ideal output which is used to describe the degree of structural damage. The obtained results demonstrate the effectiveness of the proposed approach in simultaneously improving the structural damage identification precision including the damage locating and severity.
\end{abstract}

\section{Keywords}

Damage Identification, Rotation Mode, Wavelet Singularity Theory, BP Neural Network, Genetic Algorithm

\section{Introduction}

In recent years, extensive engineering application of wavelet to structure damage identification has been reported, as it has been recognized that using such an approach yields improved damage detection effects [1]. Structure can be regarded as a mechanical system consisting of stiffness, mass and damping matrix. Once the structure is damaged, structural parameters are also changed, which leads to the change of system frequency response function and modal parame- 
ters (natural frequencies, damping and mode, etc.). Therefore, the modal parameters of a structure change can be regarded as a symbol of early damage. The damage location and degree can be determined by analyzing the transformation of these modal parameters. The damage identification problem based on the change of the structural modal parameters can be regarded as the inverse problem of structure [2] [3].

Recently, some artificial intelligence (AI) methods have been widely used in structural damage diagnosis, such as neural network (NN) and etc. The Neural network optimization method has strong robustness and nonlinear mapping ability, and the mapping relationship between input parameters and damage state is established in structural damage identification, which is widely used in fault diagnosis. The genetic algorithm is a kind of optimization method based on biological genetic and natural selection mechanism. Its advantages lie in that it does not depend on the specific domain of the problem, and does not require the continuity of the objective function and the type of the gradient information to the problem. For these reasons, NN optimization has found its ways in railway structural healthy monitoring applications.

The literature on structural damage identification used wavelet is quite extensive and the research activities in this field have focused on a wide variety of applications. As a new signal processing method, wavelet analysis has the characteristic of spatial localization. The wavelet transform of the signal at a certain point is determined by the local information in the low scale, which is determined by the local information, so it is able to analyze the singularity of the signal. Wavelet transform overcomes the problem that some characteristic signals are easily lost in the process of Fourier transform from time domain to frequency domain. In addition, it has more complete analysis functions in both the time domain and the frequency domain. It can not only perform signal vibration measurement and multi-scale analysis, but also perform signal vibration measurement and multi-scale analysis. By analyzing the time-varying properties of the system, the damage is identified, and the existence of the damage is shown directly in the wavelet transform. Fan et al. [1] proposed the wavelet method which is compared with the 2-D strain energy method (SEM) to demonstrate the proposed algorithm is superior with limited sensor data. Gokdag et al. [2] used a wavelet method based on the assumption that a damaged mode shape of a beam is approximately composed of an undamaged mode and other contributors such as measurement induced variations. Xu et al. [3] found two-dimensional directional Gaussian wavelet can characterize directional information about damage and described the operating deflection shapes can be used to locate the real-time dynamic characteristics of a plate. Le et al. [4] proposed a new method based on the similar formulation between the free decay response and the correlation function of the response of a linear mechanical system under ambient excitation.

The neural network method to identify structural damage has also made a lot of useful results. Mehrjoo et al. [5] applied a method for estimating the damage intensities of joints for truss bridge structures using a back-propagation based 
neural network. Liu et al. [6] combined the back-propagation neural network with the computational mechanics to the detection and sizing of cracks. Yam et al. [7] presented an integrated method for damage detection of composite structures using their vibration responses, wavelet transform and artificial neural networks (ANN) identification. Lu et al. [8] used an innovative neural network-based pattern analysis tool to identify damage-sensitive frequency ranges autonomously and provide detailed information such as the damage type and severity. Adeli et al. [9] applied a new dynamic time-delay fuzzy wavelet neural network model for nonparametric identification of structures using the nonlinear autoregressive moving average with exogenous inputs approach. Liu et al. [10] performed a structure damage diagnosis method combining the wavelet packet decomposition, multi-sensor feature fusion theory and neural network pattern classification. Gao et al. [11] improved wavelet threshold algorithm to eliminate the noise for vibration signals. Sahoo et al. [12] used a hybrid neuro-genetic algorithm to automate the design of neural networks for different types of structures based on the fact that the damage has an important effect on the static as well as dynamic behavior of the structure. Vakil-Baghmisheh et al. [13] proposed a fault diagnosis method based on genetic algorithms (GAs) and a model of damaged (cracked) structure. Buezas et al. [14] presented methodology based on genetic algorithms for the crack detection on damaged structural elements. Meruane, et al. [15] proposed a hybrid real-coded Genetic Algorithm with damage penalization is implemented to locate and quantify structural damage. Hao, et al. [16] applied a genetic algorithm with real number encoding to identify the structural damage by minimizing the objective function, which directly compares the changes in the measurements before and after damage.

However, fewer studies have considered the application of wavelet method and NN optimization to structure damage identification location and damage severity quantitative problems. At present, most of the identification methods are based on natural frequency and modal parameters to identify structural damage, but there are still many shortcomings in the identification efficiency, noise resistance and quantitative analysis. The wavelet analysis method can effectively determine the location of damage using wavelet singularity detection theory. But it is still needed to improve the accuracy of calculation for quantitative determination of the degree of damage. The nonlinear mapping ability of a neural network can establish the mapping relationship between the input and output parameters in the structural damage detection. In this paper, an optimized damage identification method combined the wavelet transform with neural network optimization is presented to improve the identification effects of the damage location and degree.

\section{Basic Theory of Wavelet Neural Network}

\subsection{Wavelet Singularity Identification}

If the mother wavelet $\psi(t) \in L^{2}(R)$ and $\psi(0)=0, \int_{-\infty}^{+\infty} \psi(t) \mathrm{d} t=0$, the 
wavelet function family is generated by the scale expansion and the time translation, that is, the wavelet generating function [17]:

$$
\psi_{a, b}(t)=\frac{1}{\sqrt{a}} \psi\left(\frac{t-b}{a}\right) \quad a, b \in R, a \neq 0
$$

Mother wavelet satisfying the following admissibility condition:

$$
C_{\psi}=\int_{-\infty}^{+\infty} \frac{|\hat{\psi}(\omega)|^{2}}{|\omega|} \mathrm{d} \omega<+\infty
$$

where $\hat{\psi}(\omega)$ is the Fourier transform of $\psi(t)$.

For any function or signal $f(t) \in L^{2}(R)$, function $\psi(t)$ satisfies the condition of wavelet admissibility, where $t$ denotes spatial coordinate, the continuous wavelet transform (CWT) is the inner product of the signal function with the wavelet functions:

$$
W T_{f}(a, b)=\frac{1}{\sqrt{|a|}} \int_{-\infty}^{+\infty} f(t) \psi^{*}\left(\frac{t-b}{a}\right) \mathrm{d} t=\left\langle f(t), \psi_{a, b}(t)\right\rangle
$$

where $a$ and $b$ are the scale and translation factor which reflect the frequency and time information of the signal. $W T_{f}(a, b)$ is called a wavelet coefficient for the wavelet $\psi_{a, b}(t)$.

When using the wavelet transform to detect the singularity of the function, the wavelet transform given by Equation (2) can be rewritten as a convolution product:

$$
W T_{f}(a, b)=\frac{1}{\sqrt{|a|}} \int_{-\infty}^{+\infty} f(t) \psi^{*}\left(\frac{t-b}{a}\right) \mathrm{d} t=\sqrt{|a|} f * \bar{\psi}_{|a|}(b)
$$

where $\psi^{*}(t)$ is the complex conjugate function of $\psi(t)$, and the window function $\psi_{a, b}(t)=|a|^{-1 / 2} \psi(t-b / a), \quad \bar{\psi}_{|a|}(t)=|a|^{-1} \psi^{*}(-t / a)$. Therefore, wavelet transform can be regarded as the convolution operation of the signal and filter. $\bar{\psi}_{|a|}(t)$ can be understood as a (high pass) filter.

Assumed that $\theta(t)$ is a smooth function, such as the Gauss function or the three spline wavelet. Wavelet function $\psi(t)$ is the first derivative of $\theta(t)$ :

$$
\psi(t)=\frac{\mathrm{d} \theta}{\mathrm{d} t}, \theta_{s}(t)=\frac{1}{s} \theta\left(\frac{1}{s}\right)
$$

The continuous wavelet transform of the function $f(t)$ is:

$$
W f(s, u)=s^{1 / 2}\left(f^{*} \bar{\psi}_{s}\right)(u)=s^{1 / 2} \frac{\mathrm{d}}{\mathrm{d} u}\left(f^{*} \bar{\theta}_{s}\right)(u) \quad(s>0)
$$

Equation (3) shows that wavelet transform modulus maxima $|W f(s, u)|$ is the maximum value of the first derivative after $f$ polished by $\bar{\theta}_{s}$, and the $f$ of the corresponding signal is the mutation point.

If the function is not continuous, the function has a singularity at this point, and the point is the singular point. In the detection of structural singularity, the singular points contain the information of the state of the structure. The damage of the structure will cause the smaller changes of the corresponding signal, so it can be used to determine the structural damage by the detection of the signal 
mutation point. Wavelet transform is used to detect the mutation point of the signal, and the original signal must be polished at different scales, and then the maximum or zero points of the first or second order derivative of the signal after polishing is detected. The absolute value of the wavelet coefficients at the point of the signal mutation is relatively large, that is, the singularity of the signal. The position and singularity of the singular point of the damage can be determined by the detection of the modulus maximum or zero crossing point.

\subsection{BP Neural Network Technology}

The artificial neural network has a strong ability of pattern classification and recognition, and has been widely used in structural damage detection. BP neural network is a multilayer feed-forward network with error back propagation. Its structure is simple and the algorithm is easy to implement, the system can solve learning problems in a multilayer network containing layer unit is connected with the right. The basic principle of the BP learning algorithm is the steepest descent method, the central idea is to adjust the weights to minimize the total error of the network [18]. The gradient search technique is used to minimize the error of the actual output and the expected output value of the network.

Taking the 3 layer BP network as an example, the network structure is shown in Figure 1. $x_{i}(i=1,2, \cdots, n)$ is the network input parameter; $y_{j}(j=1,2, \cdots, m)$ is the output parameter; $s_{l}(l=1,2, \cdots, p)$ is the hidden layer node. The activation function is the Sigmoid function, therefore the output of the hidden layer and the output layer are respectively [19]:

$$
\begin{aligned}
& s_{l}=g\left(\sum_{i=1}^{n} \omega_{i l} x_{i}-\theta_{l}\right)=f\left(\text { net }_{l}\right) \\
& y_{j}=g\left(\sum_{l=1}^{p} \omega_{l j} x_{l}-\theta_{j}\right)=f\left(\text { net }_{j}\right)
\end{aligned}
$$

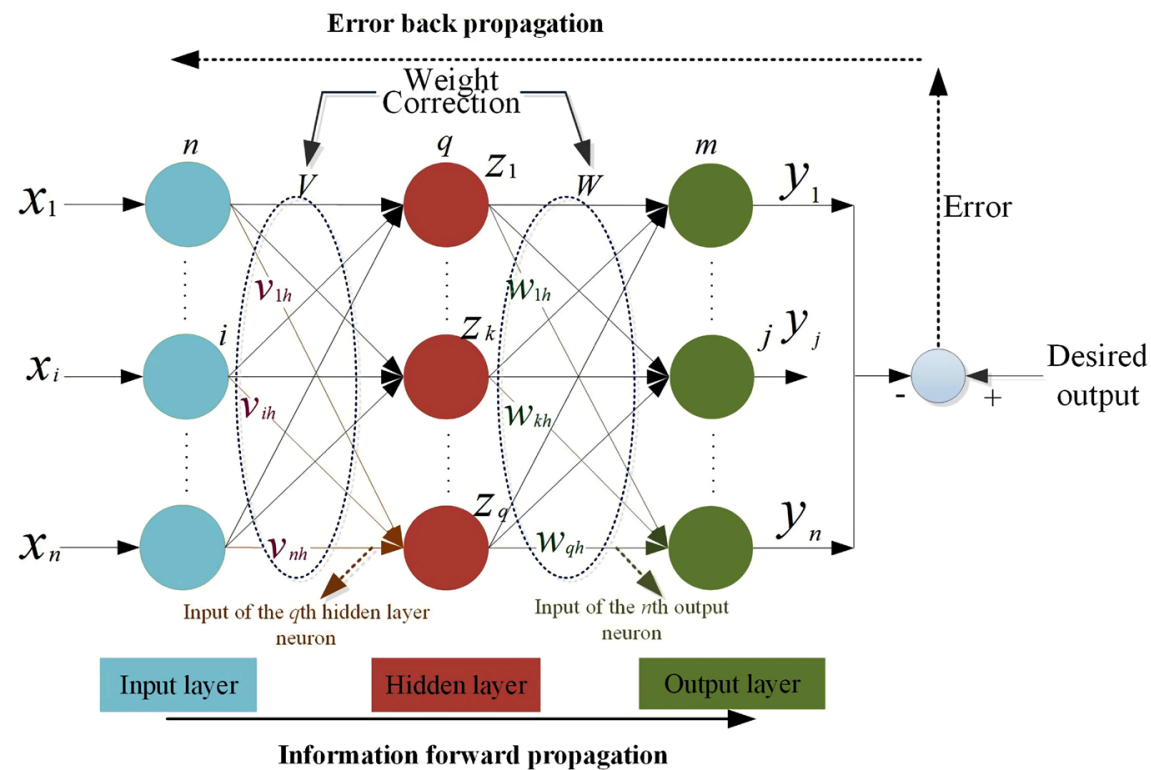

Figure 1. Structure of the BP neural network. 
where $\omega_{i l}, \omega_{l j}$ are the network weights between the input-hidden layer and the hidden-output layer; $\theta_{l}, \theta_{j}$ are the threshold of the network; $s_{l}, y_{j}$ the output of the hidden layer and the output layer, respectively.

The total error of $n$ samples can be described as follow:

$$
\begin{gathered}
E=\sum_{q=1}^{Q} E_{q} \\
E_{q}=\sum_{j=1}^{m}\left(t_{l}^{(q)}-y_{l}^{(q)}\right)^{2}
\end{gathered}
$$

where $E_{q}$ is the error of a sample, $t_{l}$ is described for the network expected output, $q$ is the number of samples.

The output error of the network is a function of the weight of each layer, and the adjustment of the weight is proportional to the negative gradient of the error, so the error $E$ can be changed by adjusting the weight. The gradient descent method is used to train the network, and the weights and thresholds are adjusted continuously until the error or the training times can meet the requirements.

$$
\begin{aligned}
\Delta \omega_{i l} & =-\frac{\partial E}{\partial \omega_{i l}}(i=1,2, \cdots, n ; l=1,2, \cdots, p) \\
\Delta \omega_{l j} & =-\frac{\partial E}{\partial \omega_{l j}}(l=1,2, \cdots, p ; j=1,2, \cdots, m)
\end{aligned}
$$

\subsection{Optimization of Neural Network Based on Genetic Algorithm}

Genetic Algorithms (GAs) is a random search algorithm based on natural selection and natural genetic mechanisms. Based on the principle of "survival of the fittest and survival of the fittest", the algorithm is a global optimization algorithm based on genetic manipulation such as selection, crossover and mutation. Genetic algorithm is used to estimate the fitness of each individual in the population by minimizing the error between the numerical simulation and the measurement frequency to realize the regional large-scale search.

Because the genetic algorithm has a fast convergence speed, high efficiency, global search and suitable for parallel processing advantages, for the optimization of BP neural network to overcome the convergence speed of BP neural network is slow, is easy to be trapped into local minima. Through the BP neural network weights and threshold encode chromosome formation, then to simulate the evolution process of nature, reproduction, crossover and mutation of a chromosome, chromosome evolution, resulting in an optimal solution to the problems on behalf of the chromosome, so as to obtain the optimization of the network weights and threshold. And then the optimized network weights and thresholds are taken as the initial values of the BP neural network weight coefficients and thresholds. The specific operation of the hybrid algorithm to optimize the network weights using a genetic algorithm is as follow [20]:

(1) Generates chromosomes and initial populations: The initial weight of the neural network is $W_{i}$, which it is equivalent to a chromosome $W_{i}=\left\{w_{1 j}, w_{2 j}, w_{3 j}, b_{1 j}, b_{2 j}, b_{3 j}, i, j=1,2, \cdots, P\right\}$. The total number of chromo- 
somes is $P$, which is population size $P$. The input layer weight $w_{1 j}$, hidden layer weight $w_{2 j}$, output layer weight $w_{3 j}$, and the corresponding node threshold $b_{1 j}, b_{2 j}, b_{3 j}$, constitute a complete set of network weights, the group of network weights as a chromosome.

(2) Objective function and fitness function: An important performance index of the BP network is the sum of the squares of errors between the actual output and the expected output of the network. The smaller the $E$, the better the performance of the network.

$$
E=\frac{1}{2} \sum_{p} \sum_{l}\left(t_{p l}-O_{p l}\right)^{2}
$$

where $t_{p l}$ is the expected output of the $p$ th output node of the th learning sample; $O_{p l}$ is the actual output of the $p$ th output node of the th learning sample. Genetic algorithm is through the fitness function to reflect the size of the individual's advantages and disadvantages, $C$ is a constant, the definition of evaluation of the merits of the fitness function of neural networks:

$$
F=C-E
$$

The error squared sum $E$ and the fitness function $F$ are functions of the weights and thresholds of the network, which is $F=f(W, \theta)$. Therefore, the objective function of genetic algorithm optimization is to find a matrix of weights $W_{0}$ and threshold matrix $\theta_{0}$, so that:

$$
F=f\left(W_{0}, \theta_{0}\right) \rightarrow \max (=C)
$$

(3) Through the selection, crossover, mutation genetic algorithm obtain the new gene values after the mutation. After several iterations, a set of weights and thresholds that minimize neural network errors are obtained, hybrid neural network genetic algorithm flow chart is shown in Figure 2.

\section{Structural Damage Identification Theory}

\subsection{Damage Identification Based on Wavelet Transform of Rotation Mode}

Mathematically, the general function is smooth or without singularity. If the function is not continuous in a certain position or in a certain order, if the function is called, the singular features or irregularities contained in the basic information of the signal must be considered. The singularity of the general signal is divided into two kinds of situations: 1) at a certain moment, the amplitude of the signal is abrupt, which causes the discontinuity of the signal. This kind of mutation is called the discontinuity point between the first class; 2) the appearance of the signal is very smooth, there is no abrupt change in amplitude, but the first order differential of the signal is abrupt and the first order differential is discontinuous, which is the second kind of discontinuity [21].

The existence of damage causes the local stiffness of the structure to change, which leads to the corresponding change of the natural frequency and mode shape of the structure. Under the general conditions, the structure damage location 


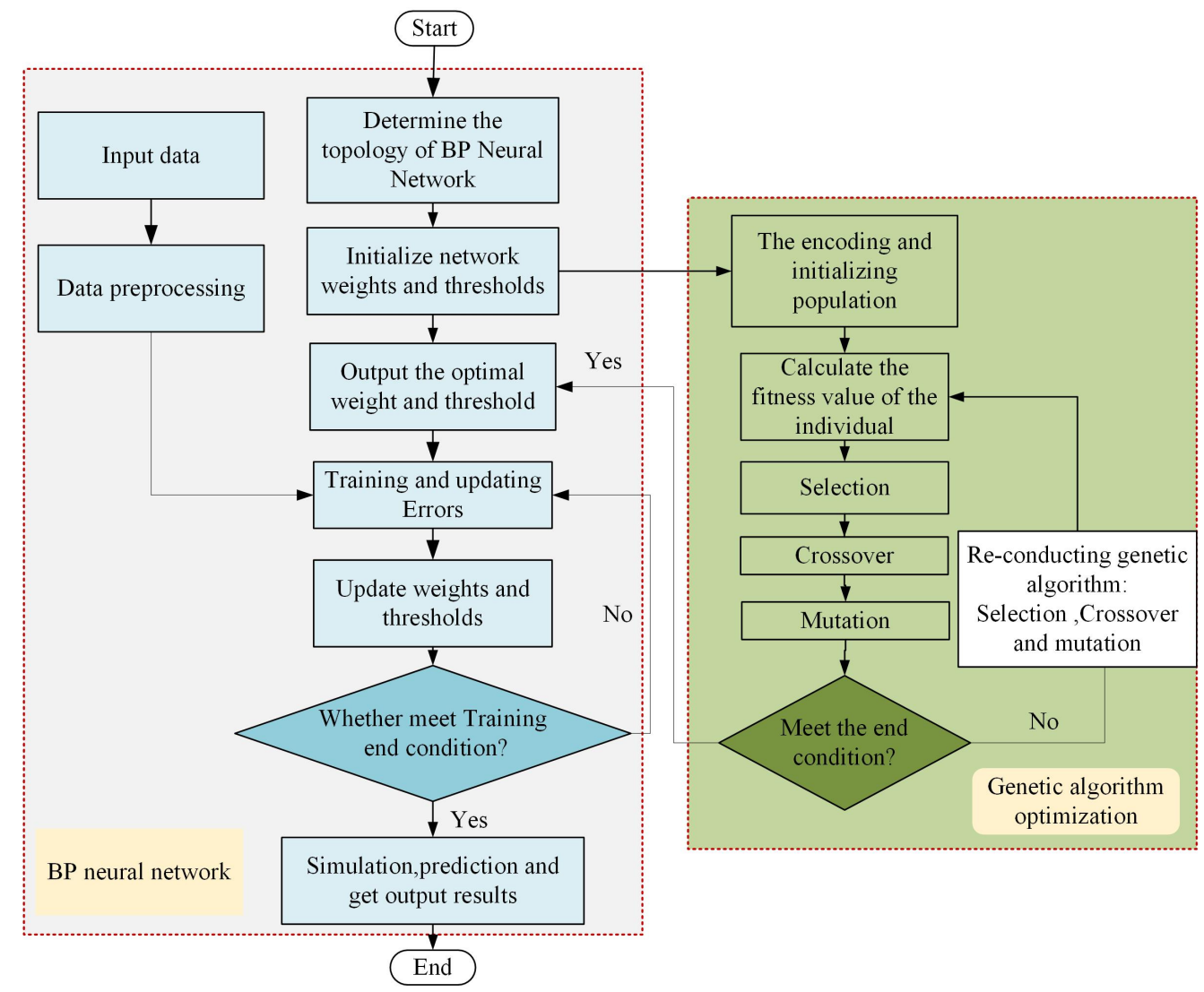

Figure 2. Hybrid neural genetic algorithm flow chart.

can be identified by the modulus maxima of the wavelet coefficient map after the structure of the basic mode of the wavelet transform. But the wavelet coefficient line is not smooth enough. In particular, many irregular processes are often produced near the end of the beam and the support, and the discrimination of the damage location is disturbed.

When the section of the beam is damaged, the stiffness of the section of the damaged structure is reduced. The bending stiffness $E I$ is only related to the elastic modulus $E$ of the material used in the structure and the geometrical properties of the section. At this point, the decrease of the section stiffness of the beam is mainly the decrease of the $E$ value of the material. The stiffness of the left and right sides of the cross-section of a changed, $E I\left(v^{+}\right) \neq E I\left(v^{-}\right)$. But the structure still should satisfy the condition of deformation compatibility and internal force balance [22].

Vertical displacement:

$$
w\left(v^{+}\right)=w\left(v^{-}\right)
$$

Rotation mode:

$$
\frac{\mathrm{d} w\left(v^{+}\right)}{\mathrm{d} x}=\frac{\mathrm{d} w\left(v^{-}\right)}{\mathrm{d} x}
$$

Bending moment: 


$$
E I\left(v^{+}\right)\left(\frac{\mathrm{d}^{2} w\left(v^{+}\right)}{\mathrm{d} x^{2}}\right)=E I\left(v^{-}\right)\left(\frac{\mathrm{d}^{2} w\left(v^{-}\right)}{\mathrm{d} x^{2}}\right)
$$

Shear force:

$$
E I\left(v^{+}\right)\left(\frac{\mathrm{d}^{3} w\left(v^{+}\right)}{\mathrm{d} x^{3}}\right)=E I\left(v^{-}\right)\left(\frac{\mathrm{d}^{3} w\left(v^{-}\right)}{\mathrm{d} x^{3}}\right)
$$

It can be known that only the vertical displacement and its first derivative (Rotation mode) are continuous, but $\frac{\mathrm{d}^{2} w\left(v^{+}\right)}{\mathrm{d} x^{2}} \neq \frac{\mathrm{d}^{2} w\left(v^{-}\right)}{\mathrm{d} x^{2}}$. The singularity of the two order derivative is different, which can be used to identify the damage of the structure by using the rotation model.

In the detection of structural singularity, the damage of the structure can cause the micro mutation of the signal, which is called the singularity point. Compared with the normal signal, the singular point contains the information of the structure state change, so the detection of the signal mutation points can be used for structural damage identification. There are two methods to deal with the singular signal, one is based on Fourier transform, the other is based on wavelet transform.

The Fourier transform has been a basic tool to study the singularity of function. It is through the function of the Fourier transform of the speed of the zero to infer whether the function of singularity and singularity of the strength. However, due to the lack of spatial locality of the Fourier transform, the Fourier transform of the function can only determine the global nature of the singularity, and it is difficult to determine the distribution of the singular points in space. It is difficult to determine the position of each singular point and the strength of singularity when the function has many singular points.

Because the wavelet transform can map any signal to a set of basis functions which are composed of a mother wavelet expansion and translation, it has the characteristic of spatial localization. The wavelet transform of the signal at a certain point is determined by the local information in the low scale. It can achieve a reasonable separation of the signal at different time and different frequency bands without losing the original information. Therefore, it is more effective to use the wavelet transform to analyze the singularity and the singularity of the signal.

The selection of wavelet basis functions is directly related to the effectiveness of the analytical results when the wavelet transform is dealing with the singular signal. In order to ensure the accurate identification of the signal of the mutation point, in the choice of wavelet basis, mainly to meet the fixed interval of the tight support and enough to disappear. When selecting the scale, the scale range is $0.25 \leq s \leq 7$, so that it can effectively eliminate the influence of the noise and find the singular point. In this paper, we use the two order derivative Mexh wavelet of Guass function: 


$$
\psi(t)=\frac{2}{\sqrt{3 \sqrt{\pi}}}\left(1-t^{2}\right) \mathrm{e}^{-t^{2} / 2}
$$

For the mother wavelet function, the continuous wavelet transform is performed on the corner of the damaged beam. The modulus maxima can occur in the wavelet coefficients at the change of stiffness, which can determine the location of the damaged area.

\subsection{The Establishment of BP Neural Network}

The greater is the fitness function value, the smaller is the error between the network predictive value and the actual value. Table 1 lists the basic parameters of the genetic algorithm. Neural network method has a strong ability of nonlinear mapping and pattern recognition, as long as there is some kind of rule between the data, using neural network can describe the law with good accuracy. The neural network establishes the mapping relationship between input and output, and the hidden layer really performs this kind of relationship. Hidden layer neurons number selection on the convergence speed is very important, increase the implicit layer number can further reduce the training error and improve the precision of the simulation, but will also make the complex network, thereby increasing the training time of the network. The study showed that the three layers BP neural network can simulate the nonlinear relationship between any input and output. The complexity depends on the number of neurons in the hidden layer, and the number of neurons in each layer varies according to the specific problem. In this paper, the three layer BP network is used to input the parameters of the model, the maximum value of the wavelet coefficient modulus of the front $\mathrm{n}$ mode of the damaged beam, and the output parameters are the degree of damage. So the network structure is established for 4-8-1 (single point damage), as shown in Figure 3. The output layer neuron uses the pure linear

Table 1. Basic elements of genetic algorithm.

\begin{tabular}{ccc}
\hline No. & Element & Values \\
\hline 1 & Population size & 50 \\
2 & Generation & 100 \\
3 & Chromosome length & 92 \\
4 & Selection probability $\left(p_{x}\right)$ & 0.95 \\
5 & Crossover probability $\left(p_{c}\right)$ & 0.4 \\
6 & Mutation probability & 0.05 \\
\hline
\end{tabular}
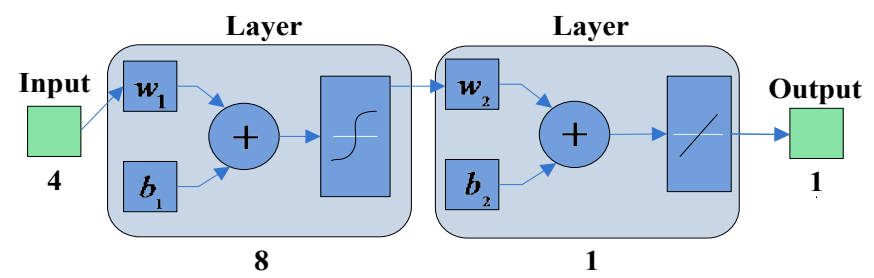

Figure 3. Three-layer BP network utilized in this study. 
transformation function, and then sets up the corresponding learning rate and the training goal, and so on.

\section{Numerical Example}

In order to verify the validity and accuracy of the algorithm, the three-span continuous beam is used as an example to simulate the structural damage by reducing the bending stiffness of the element. Mexh wavelet is used to carry out the continuous wavelet transform of the structure rotation mode, and the damage location of the structure is determined. The input sample of BP neural network is the wavelet transform modulus maxima of the rotation mode, the network output is the structure damage degree. Firstly, the finite element model of the continuous beam is established, and the modal parameters of the structure are obtained. Then, based on the wavelet singularity detection theory, the wavelet coefficients are obtained by using the wavelet transform, and the wavelet coefficients are obtained. The damage location is judged according to the modulus maxima of wavelet coefficients. Finally, the wavelet coefficients modulus maxima of the wavelet coefficients of the multiple scales are used as the input parameters of the BP neural network. The output result is the degree of structural damage, while introducing genetic algorithm to optimize the parameters of the neural network structure, obtained the corresponding network forecasting value. The three-span continuous beam finite element model is established in software ANSYS. The three-span continuous beam structure model can be shown in Figure 4.

\subsection{Single Damage Event}

The assumption is that there is damage in the span of the continuous beam (i.e., element 50). Figure 5 and Figure 6 respectively show the continuous beam damage before and after the basic mode of vibration mode and the first order rotation mode.

The fundamental mode of the continuous beam is wavelet transform, as shown in Figure 7. It can be seen that there is no obvious singularity characteristic of the basic modal parameters after the damage. Due to the near the bearing is boundary condition effects, the presence of vertical constraint and the deflection approximate to zero and around the corner there is a marked difference. Therefore, the use of basic mode shape wavelet transform can not accurately

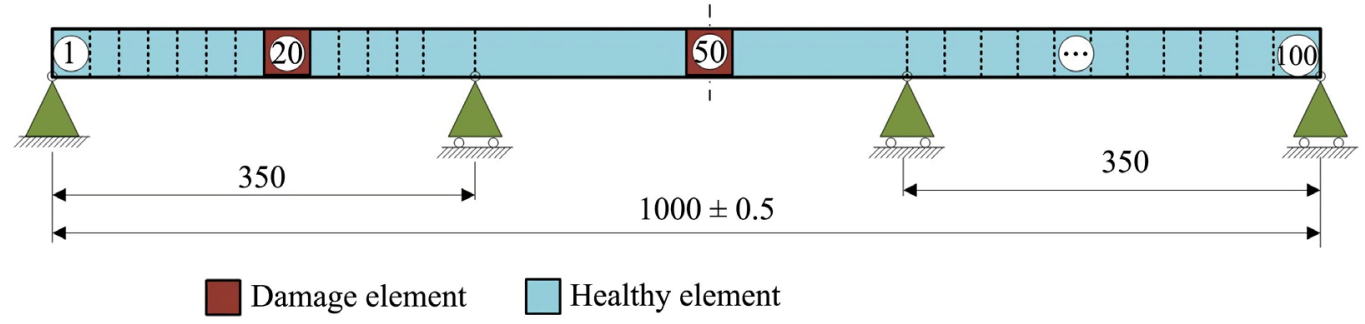

Figure 4. The three-span continuous beam structure. 


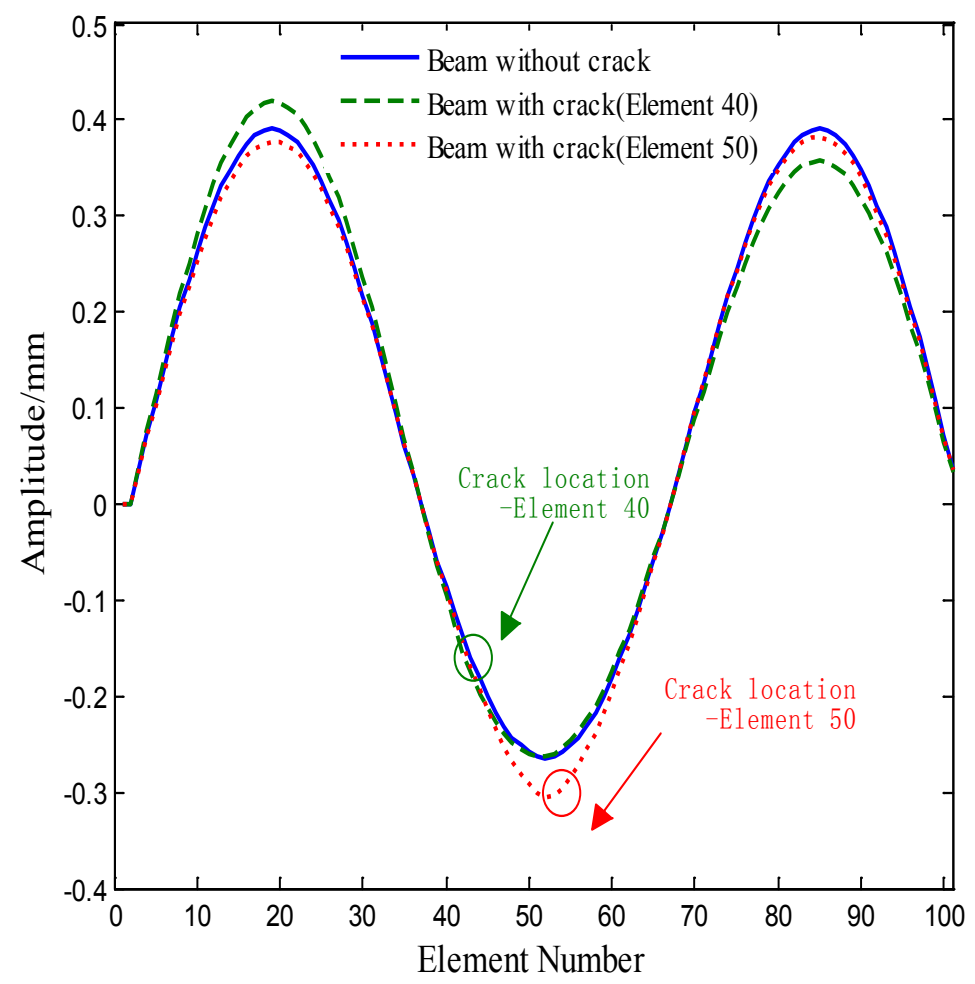

Figure 5. The basic mode shapes of the continuous beam before and after the damage.

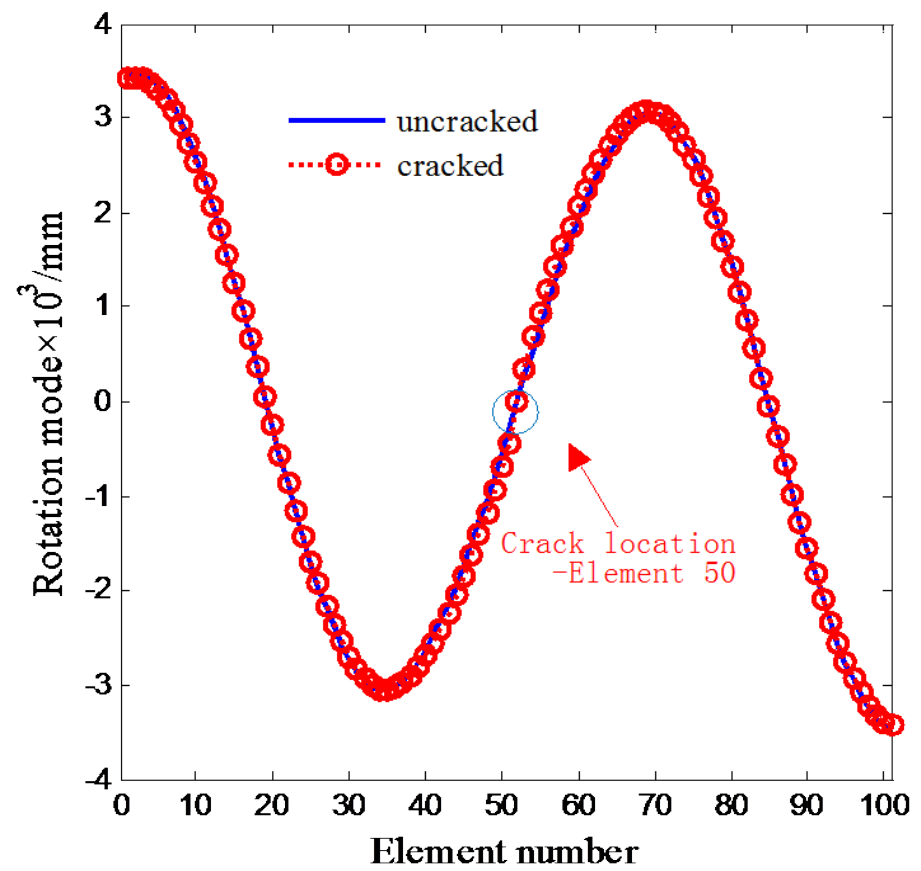

Figure 6. The first rotation mode of the continuous beam.

identify damage. The Mexh wavelet transform is used to carry out the continuous wavelet transform of the corner mode of the damaged beam, and the multi-scale wavelet coefficients and the gray scale map are obtained as shown in Figure 8 and Figure 9. It can be seen from the figure that the modulus maxima 


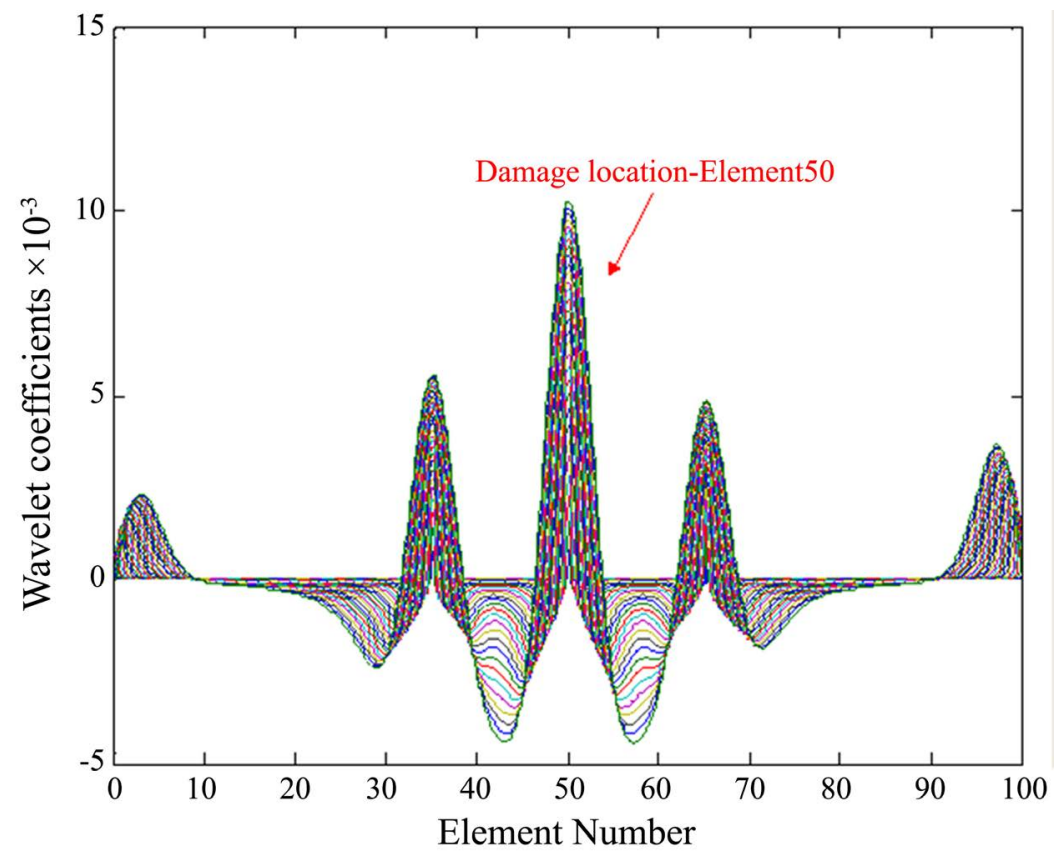

Figure 7. The multi-scale wavelet coefficients of displacement mode.

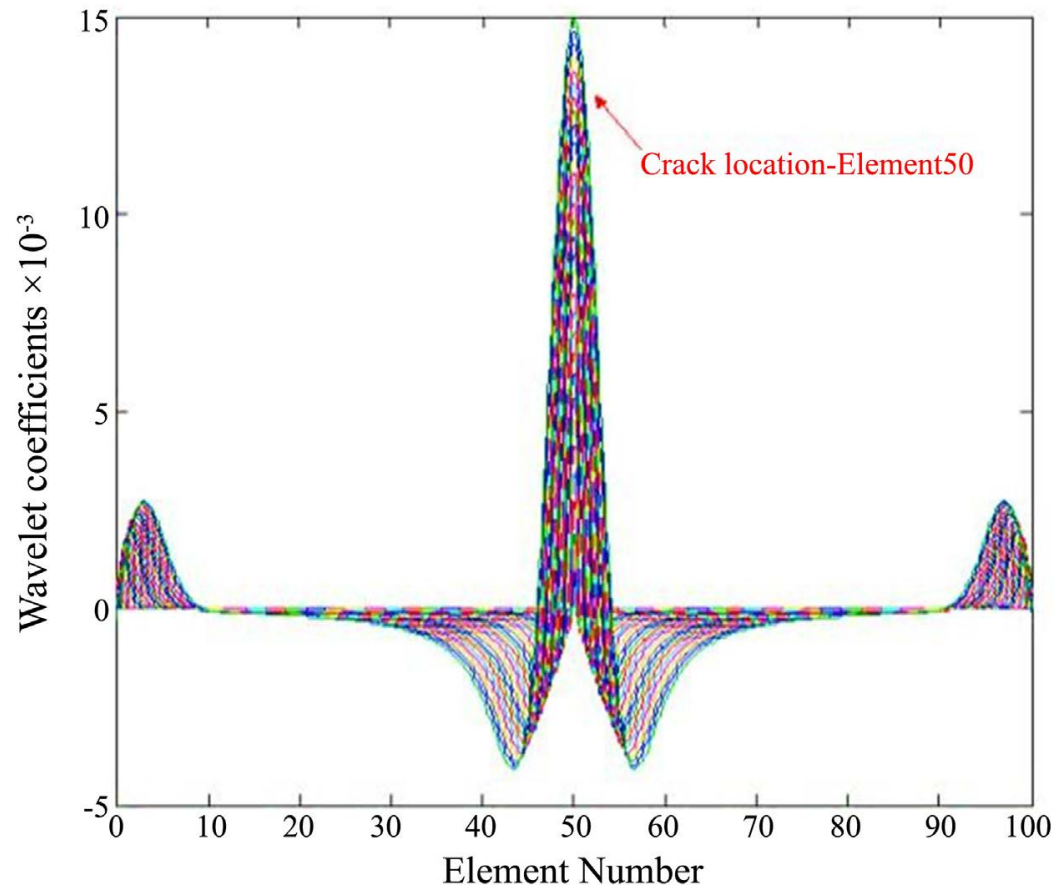

Figure 8. The multi-scale wavelet coefficients of rotation mode.

of the wavelet coefficients appear in element 50, so the damage location can be judged in the span of the beam.

In order to prepare the learning data samples of the network, the damage degree of the continuous beam damage location (element 50) is simulated, and the damage conditions of the groups are in a total of 16 groups. Based on the 4 layers BP neural network, the wavelet transform modulus maxima of the first three 


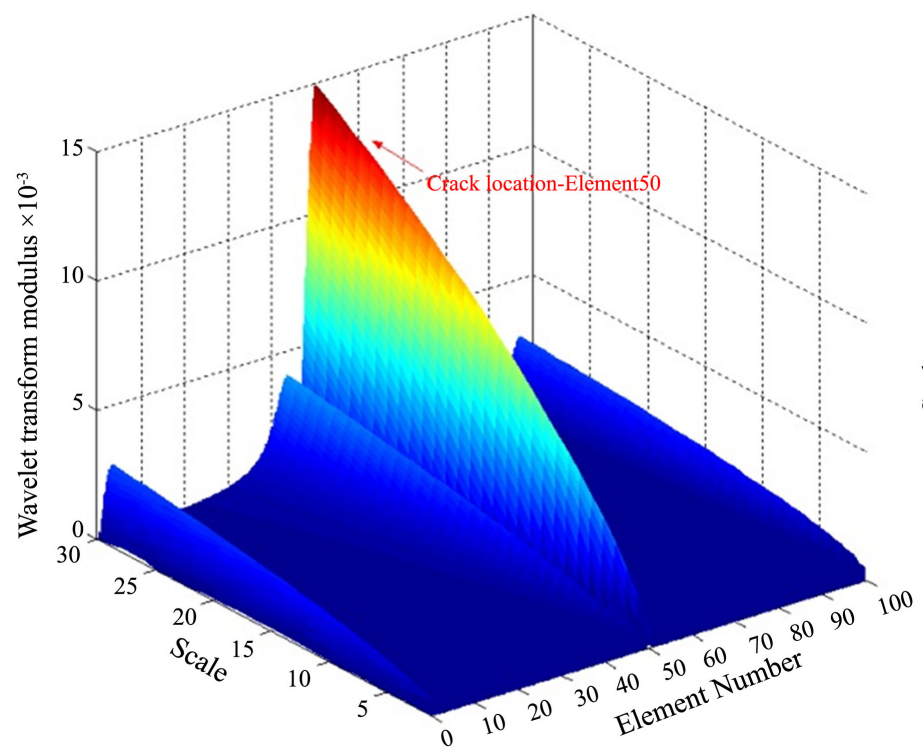

(a)

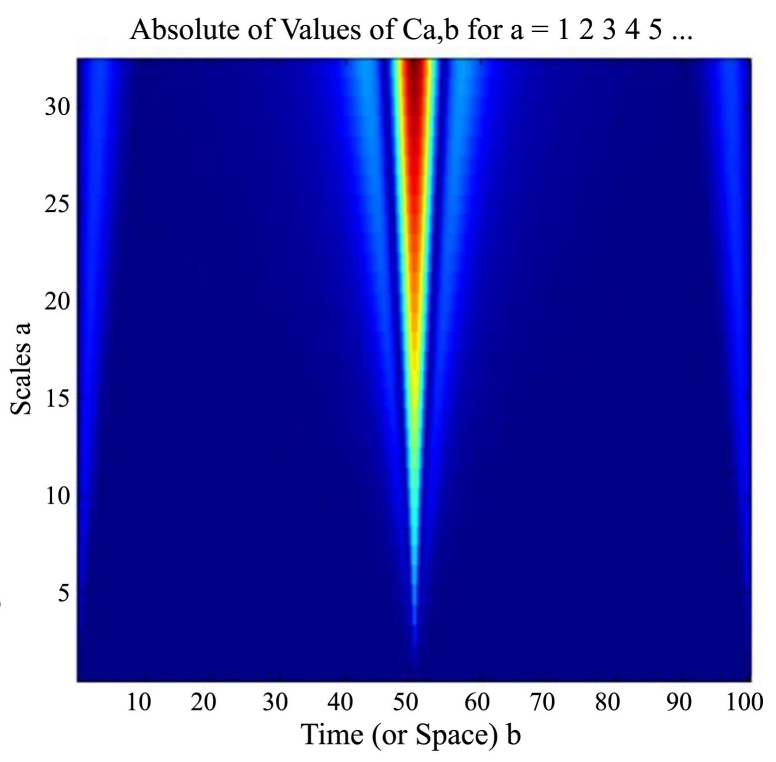

(b)

Figure 9. Wavelet transform modulus of first rotation mode. (a) 3D view; (b) top view.

scales are used as the input parameters of the neural network, the output parameter is the damage degree of the element, and the network structure is 4-8-1. Then, the wavelet transforms maxima of the 7 kinds of damage cases (damage degree) of the element 50 in the first 4 scales are used as the network test data. As shown in Table 2, the corresponding network test results and relative error can be obtained, and then the mean value of the network prediction error of the damage degree of the continuous beam (element 50 ) is calculated.

\subsection{Multiple Damage Event}

In order to simulate the multi loss working conditions, the damage is assumed to exist in the element 20 and element 50 in the continuous beam. Figure 10 is the Mexh wavelet transform coefficients and the gray scale of the damaged beam, which can be clearly defined in the figure. There are two damages in the continuous beam. Damage location is determined, and then uses the BP neural network to identify the degree of damage. The damage degree of the element 20 and element 50 were $\alpha=10 \%, 20 \%, 30 \%, 40 \%$ and $\beta=10,20 \%, 80 \%$. A total of 32 groups of network training samples. Similarly, the wavelet coefficients modulus maxima of the damaged beam in the first 4 scales are used as the input parameters of the network, and the output is two damage state. The neural network structure is 4-10-2. Table 3 lists the wavelet transform modulus maxima and the corresponding BP network test results for the different damage degree of the multiple damaged beams.

As can be seen from Table 3, the BP network can be constructed to identify the damage degree of the beam at the same time, and the relative error is small. Element 20 and Element 50 damage error graph and the average value of element 20 , the element of 50 damage prediction errors were $0.22 \%$ and $0.18 \%$. 


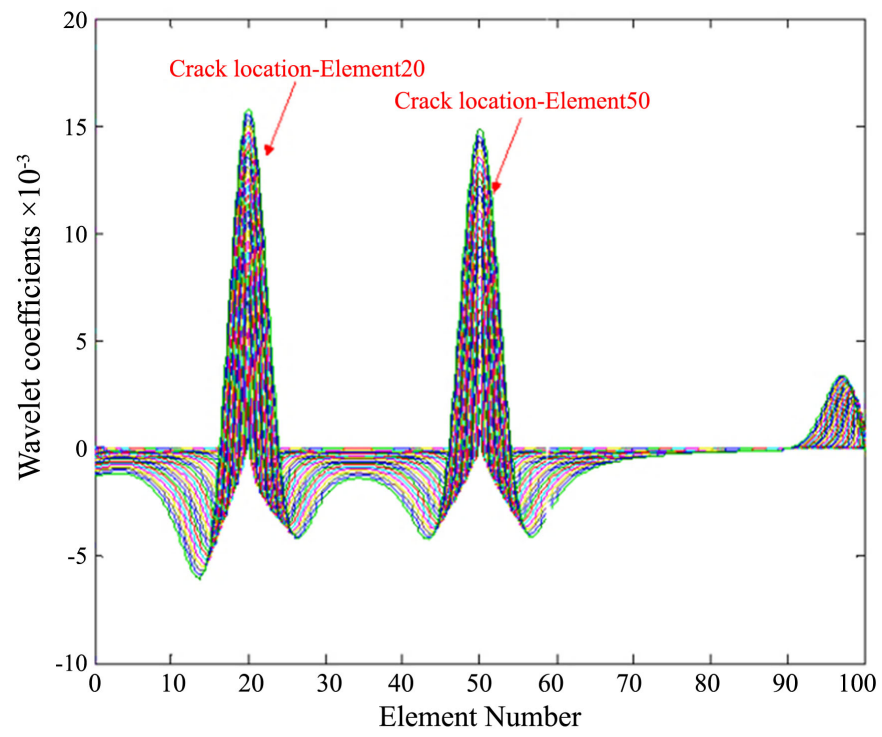

(a)

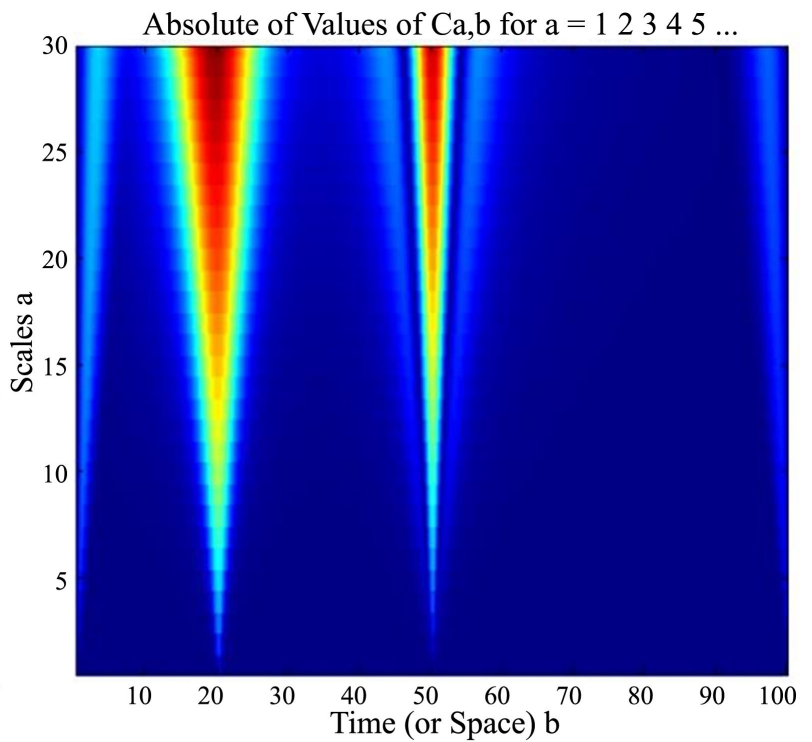

(b)

Figure 10. The multi-scale wavelet coefficients and Gray scale of rotation mode. (a) wavelet coefficients; (b) Gray scale.

Table 2. Different damage degree of the test results of element 50.

\begin{tabular}{ccccccc}
\hline \multirow{2}{*}{$\begin{array}{c}\text { Decline rate of } \\
\text { flexural rigidity/\% } \%\end{array}$} & \multicolumn{3}{c}{ Maximum of wavelet coefficients } & \multicolumn{2}{c}{ The results of BP neural network testing } \\
\cline { 2 - 7 } & 1 scale & 2 scale & 3 scale & 4 scale & Predicted value & Relative error/\% \\
\hline 15 & 1.572 & 2.363 & 3.260 & 4.484 & 15.097 & 0.65 \\
25 & 1.585 & 2.366 & 3.265 & 4.504 & 25.037 & 0.15 \\
35 & 1.602 & 2.370 & 3.272 & 4.530 & 35.087 & 0.25 \\
45 & 1.624 & 2.375 & 3.281 & 4.565 & 45.025 & 0.06 \\
55 & 1.656 & 2.383 & 3.293 & 4.615 & 55.057 & 0.10 \\
65 & 1.704 & 2.394 & 3.311 & 4.691 & 64.966 & -0.05 \\
75 & 1.787 & 2.413 & 3.342 & 4.820 & 74.978 & -0.03 \\
\hline
\end{tabular}

Table 3. Comparison of the identification results of element 20 and 50.

\begin{tabular}{ccccccccccc}
\hline $\begin{array}{c}\text { Locations vs } \\
\text { degrees of damage }\end{array}$ & \multicolumn{2}{c}{ Maximum of wavelet coefficients } & \multicolumn{3}{c}{$\begin{array}{c}\text { The results of BP } \\
\text { neural network testing }\end{array}$} \\
\hline Element 20 Element 50 & 1 scale & 2 scale & 3 scale & 4 scale & Predicted value & Relative error/\% \\
\hline $10 \%$ & $10 \%$ & 1.561 & 2.483 & 3.438 & 4.575 & 10.032 & 10.013 & 0.32 & 0.13 \\
$10 \%$ & $20 \%$ & 1.571 & 2.480 & 3.434 & 4.569 & 9.985 & 19.956 & -0.15 & -0.22 \\
$20 \%$ & $30 \%$ & 1.575 & 2.490 & 3.447 & 4.601 & 20.093 & 30.112 & 0.46 & 0.37 \\
$20 \%$ & $40 \%$ & 1.595 & 2.484 & 3.439 & 4.591 & 19.926 & 39.993 & -0.37 & -0.02 \\
$30 \%$ & $50 \%$ & 1.60 & 2.493 & 3.452 & 4.626 & 30.012 & 49.883 & 0.04 & -0.23 \\
$30 \%$ & $60 \%$ & 1.648 & 2.480 & 3.434 & 4.606 & 30.044 & 60.022 & 0.15 & 0.04 \\
$40 \%$ & $70 \%$ & 1.695 & 2.480 & 3.436 & 4.634 & 39.976 & 70.069 & -0.06 & 0.10 \\
$40 \%$ & $80 \%$ & 1.813 & 2.439 & 3.381 & 4.678 & 39.980 & 79.992 & -0.05 & -0.01 \\
\hline
\end{tabular}


Therefore, the BP NN network prediction value and element damage are closer and the relative error is smaller.

\subsection{Results Analysis and Discussion}

The numerical calculation example shows that the structural damage identification method based on the rotation mode is not only more sensitive to damage, but also avoids the singularity caused by the effect of the boundary condition of the beam structure. Considering the wavelet damage identification method of BP neural network, it is easier to locate the damage based on the singular point than the general wavelet coefficient identification method. And the detection effect of damage degree is better. The identification range of this method includes single or multiple damaged structures. Its position recognition is mainly determined by continuous wavelet transform and rotation mode. For the structural rotation mode, the maximum value of the wavelet transform modulus is regarded as the input parameter of the BP network, and the single damage average value (Damage element 50 ) of the damage prediction error is $0.22 \%$. More importantly, the average predictions of multiple damages (Damage element 20 and 50) to the degree of damage error are $0.18 \%$ and $0.22 \%$, respectively. The results show that the proposed optimization method can not only accurately locate the damage location, but also identify the degree of damage with higher accuracy.

\section{Conclusions}

In this paper, combining continuous wavelet transform and neural network, an optimization method for structural damage identification based on rotation mode and wavelet neural network is proposed. This method can significantly improve the calculation efficiency of damage identification location and degree, so as to avoid or reduce detection errors to the greatest extent. The results also show some other research conclusions.

The combination of the proposed wavelet and neural network optimization method can provide a valuable tool in the damage identification of the health monitoring of a variety of railway vehicle structures and infrastructures, which is beneficial to the fault diagnosis and monitoring of the vehicle status in a complex load environment. Considering that the practicability of this neural network optimization tool is enhanced by the genetic algorithm optimization theory, the theory makes the analysis equally applicable to other structures. Therefore, the extension of this research is a very meaningful research topic. It not only combines the local characteristics of wavelet with the self-learning characteristics of neural network, but also can be applied to engineering practice.

\section{Acknowledgements}

The authors wish to thank the teachers and students who participated in the research team on Load Identification and Damage Identification for their contributions. The work has been financed by the National Natural Science Founda- 
tion of China (51775456), and the Self-developed Research Project of the State Key Lab. of Traction Power (2019TPL T03).

\section{Conflicts of Interest}

The authors declare no conflicts of interest regarding the publication of this paper.

\section{References}

[1] Fan, W. and Qiao, P.Z. (2009) A 2-D Continuous Wavelet Transform of Mode Shape Data for Damage Detection Of Plate Structures. International Journal of Solids and Structures, 46, 4379-4395. https://doi.org/10.1016/j.ijsolstr.2009.08.022

[2] Gkdağ, H. and Kopmaz, O. (2009) A New Damage Detection Approach FOR Beam-Type Structures Based on the Combination of Continuous and Discrete Wavelet Transforms. Journal of Sound and Vibration, 324, 1158-1180. https://doi.org/10.1016/j.jsv.2009.02.030

[3] Xu, W., Radzieński, M., Ostachowicz, W. and Cao, M. (2013) Damage Detection in Plates Using Two-Dimensional Directional Gaussian Wavelets and laser Scanned Operating Deflection Shapes. Structural Health Monitoring, 12, 457-468. https://doi.org/10.1177/1475921713492365

[4] Le, T.-P. and Paultre, P. (2012) Modal Identification Based on Continuous Wavelet Transform and Ambient Excitation Tests. Journal of Sound and Vibration, 331, 2023-2037. https://doi.org/10.1016/j.jsv.2012.01.018

[5] Mehrjoo, M., Khaji, N., Moharrmi, H. and Bahreininejad, A. (2008). Damage Detection of Truss Bridge Joints Using Artificial Neural Networks. Expert Systems with Applications, 35, 1122-1131. https://doi.org/10.1016/j.eswa.2007.08.008

[6] Liu, S.W., Huang, J.H., Sung, J.C. and Lee, C.C. (2002) Detection of Cracks Using Neural Networks and Computational Mechanics. Computer Methods in Applied Mechanics and Engineering, 191, 2831-2845. https://doi.org/10.1016/S0045-7825(02)00221-9

[7] Yam, L.H., Yan, Y.J. and Jiang, J.S. (2003). Vibration-Based Damage Detection for Composite Structures Using Wavelet Transform and Neural Network Identification. Composite Structures, 60, 403-412. https://doi.org/10.1016/S0263-8223(03)00023-0

[8] Lu, Y., Ye, L., Su, Z.Q. and Yang, C.H. (2008) Quantitative Assessment of Through-Thickness Crack Size Based on Lamb Wave Scattering in Aluminium Plates. Ndt \& E International, 41, 59-68. https://doi.org/10.1016/j.ndteint.2007.07.003

[9] Adeli, H. and Jiang, X. (2006) Dynamic Fuzzy Wavelet Neural Network Model for Structural System Identification. Journal of Structural Engineering, 132, 102-111. https://doi.org/10.1061/(ASCE)0733-9445(2006)132:1(102)

[10] Liu, Y.Y., Ju, Y.F., Duan, C.D. and Zhao, X.F. (2011) Structure Damage Diagnosis Using Neural Network and Feature Fusion. Engineering Applications of Artificial Intelligence, 24, 87-92. https://doi.org/10.1016/j.engappai.2010.08.011

[11] Gao, Z.-F. and Chen, X.-J. (2011) Structure Data Processing and Damage Identification Based on Wavelet and Artificial Neural Network. Research Journal of Applied Sciences, Engineering and Technology, 3, 1203-1208.

[12] Sahoo, B. and Maity, D. (2007) Damage Assessment of Structures Using Hybrid 
Neuro-Genetic Algorithm. Applied Soft Computing, 7, 89-104. https://doi.org/10.1016/j.asoc.2005.04.001

[13] Vakil-baghmisheh, M.T., Peimani, M., Sadeghi, M.H. and Ettefagh, M.M. (2008) Crack Detection in Beam-Like Structures Using Genetic Algorithms. Applied Soft Computing, 8, 1150-1160. https://doi.org/10.1016/j.asoc.2007.10.003

[14] Buezas, F.S., Rosales, M.B. and Filipich, C.P. (2011) Damage Detection with Genetic Algorithms Taking into Account a Crack Contact Model. Engineering Fracture Mechanics, 78, 695-712. https://doi.org/10.1016/j.engfracmech.2010.11.008

[15] Meruane, V. and Heylen, W. (2011) An Hybrid Real Genetic Algorithm to Detect Structural Damage Using Modal Properties. Mechanical Systems and Signal Processing, 25, 1559-1573. https://doi.org/10.1016/j.ymssp.2010.11.020

[16] Hao, H. and Xia, Y. (2002) Vibration-Based Damage Detection of Structures by Genetic Algorithm. Journal of Computing in Civil Engineering, 16, 222-229. https://doi.org/10.1061/(ASCE)0887-3801(2002)16:3(222)

[17] Miao, B.R., Yang, S.W., Wang M.Y., Jiang, C.Y., Peng, Q.M. and Luo Y.X. (2020) Comparison of Various Structural Damage Identification Methods Using Vibration Response. Journal of Vibration Engineering, 33, 724-733. (in Chinese)

[18] Yang, C. and Wang, Z. (2010) Application Research on Genetic Algorithm and BP Neural Network in Motor Fault Diagnosis. Noise and Vibration Control, 5, 153-156. (in Chinese)

[19] Ren, Q.W., Qiu, Y. and Ye, H.J. (2004) Structural Damage Detection Based on Wavelet Transform and Neural Network. Journal of Hohai University: Natural Sciences, 32, 295-299. (in Chinese)

[20] Wu, D.S. and Liang, L. (2004) A Strategy of Optimizing Neural Networks by Genetic Algorithm and Its Application on Credit Scoring. Chinese Journal of Management Science, 1. (in Chinese)

[21] Guan, D.Q. and Jiang, X. (2007) Damage Identification of Continuous Beam Based on Wavelet Transform of Rotation Mode. Journal of Changsha University of Science \& Technology (Natural Science), 4. (in Chinese)

[22] Hong, J.C., Kim, Y.Y., Lee, H.C. and Lee, Y.W. (2002) Damage Detection Using the Lipschitz Exponent Estimated by the Wavelet Transform: Applications to Vibration Modes of a Beam. International Journal of Solids and Structures, 39, 1803-1816. https://doi.org/10.1016/S0020-7683(01)00279-7 